\title{
Cultural Mistrust, Conspiracy Theories and Attitudes towards HIV Testing Among African Americans \\ Kelsey Ball*
}

Howard University, DC, USA

\begin{abstract}
Background: With respect to the HIV epidemic, research has shown that health behaviors are affected by a number of attitudinal factors such as cultural mistrust, medical mistrust and conspiracy beliefs. While it is clear that these variables have a deleterious effect on health behaviors, the literature fails to explore how these factors uniquely influence and predict an individual's attitudes and overall willingness to receive HIV testing. Furthermore, cognitive factors such as the need for cognitive closure have received little attention with regard to HIV testing attitudes.
\end{abstract}

Objective: The aim of this study was to determine inter-correlations between cultural mistrust, medical mistrust, HIV conspiracy theories and the need for cognitive closure and to explore the extent to which these variables predict attitudes towards HIV testing.

Methods: This study consisted of 34 Black males and 84 Black females $(n=118)$ between the ages of 16-67 years ( $\mu=26.8$ years) recruited from a Historically Black College/University (HBCU), a community college, as well as churches and local health forums in the Washington, D.C. area.

Results: Results of the inter-correlations were mixed and regression analyses revealed that cultural mistrust, medical mistrust, HIV conspiracy beliefs and the need for cognitive closure were not significant predictors of attitudes towards HIV testing in these populations.

Conclusion: The results are discussed from a psychosocial perspective and implications and future directions for public health, psychotherapy and clinical work are considered.

Keywords: Mistrust; Culture; HIV; Conspiracy; Attitude; African American

\section{Introduction}

Worldwide, over 34 million people are currently living with HIV/AIDS and more than 25 million have already died of AIDS [1]. Individuals who self-identify as Black or African American are affected by HIV more than any other racial/ethnic group [2], and both past and present research has indicated that HIV is spreading faster among African Americans than any other racial group [3]. Although African Americans comprise about $12 \%$ of the U.S. population, in $2011,43 \%$ of people living with HIV were Black and in 2012, 47\% of all individuals who received an HIV diagnosis were Black [2]. Furthermore, research has shown that an estimated 1 in 32 African American women and 1 in 16 African American men will acquire HIV in their lifetimes [4]. Despite findings that rates of infection are on the decline [5], decades of sentinel surveillance studies consistently establish that African Americans have disproportionately higher rates of HIV [6]. These data clearly demonstrate the need for implementation of interventions and public health strategies to reduce HIV disparities in the Black community.

Due to the number of medical issues that disproportionately affect African Americans, health promotion and disease prevention have become significant areas of study within this population. HIV research, in particular, has received a great deal of attention due to the destructive cognitive and attitudinal factors that are believed to promote the transmission of the virus.

A history of racism and discrimination, in addition to unethical medical experimentation of African Americans, has led to feelings of vulnerability, suspicion and mistrust among certain segments of the Black community [7]. Cultural mistrust has been defined as a tendency to distrust Whites based upon a legacy of direct or vicarious exposure to racism or unfair treatment by Whites [8]. Others have conceptualized such mistrust as a "racism reaction" that has evolved as a defensive mechanism [9]. While cultural mistrust appears to stem from a history of racism and oppression, research has indicated that medical mistrust among minorities, particularly African Americans, stemmed from various historical events, including the Tuskegee Syphilis Study $[10,11]$ and the well-documented medical experimentation on slaves [12]. Regardless of their origin, it is clear that cultural mistrust and medical mistrust have resulted in a number of health-related conspiracy theories, specifically with respect to HIV, which serve to perpetuate racial health disparities [13].

A growing body of literature lends support to the notion that cultural mistrust, medical mistrust and HIV conspiracy beliefs play a role in HIV-related health disparities. For example, as a result of cultural mistrust, African Americans with HIV have lower retention and engagement in care compared to Whites with HIV [14]. Furthermore, African American men's lower levels of health care involvement, including access to and engagement in routine health care, has been directly associated with medical mistrust [15]. In a study by Ross et al., a significant correlation was found between African American males' HIV conspiracy theory beliefs and condom use, such

*Corresponding author: Kelsey Ball, Howard University, DC, USA, Tel: 440506-4911; E-mail: kelsey.ball@bison.howard.edu

Received June 10, 2016; Accepted July 12, 2016; Published July 19, 2016

Citation: Ball K (2016) Cultural Mistrust, Conspiracy Theories and Attitudes towards HIV Testing Among African Americans. J AIDS Clin Res 7: 602. doi:10.4172/21556113.1000602

Copyright: (C) 2016 Ball K. This is an open-access article distributed under the terms of the Creative Commons Attribution License, which permits unrestricted use, distribution, and reproduction in any medium, provided the original author and source are credited. 
that those who held conspiracy beliefs were less likely to use condoms [16]. Similar findings were reported in a longitudinal study by Bogart et al.: the presence of HIV conspiracy beliefs was a significant predictor of unprotected intercourse with HIV-positive or unknown sero-status partners [10].

While it is clear that cultural mistrust, medical mistrust, and conspiracy theories play a significant role in the HIV/AIDS epidemic, research is limited with regard to more specific aspects of HIV detection, namely, HIV testing. As outlined, there is a large body of research surrounding the role of cultural mistrust, medical mistrust and conspiracy theories on negative health behaviors among African Americans; however, research exploring the role of these factors on attitudes towards HIV testing is surprisingly limited and appears to focus primarily on African populations [17].

Although mistrust and conspiracy beliefs play a role in one's health care decisions and behaviors, it is also likely that cognitive differences and dispositional factors also play a role. The need for cognitive closure (NFCC) is defined as a desire for "an answer on a given topic, any answer...compared to confusion and ambiguity" [18]. To date, the role of NFCC with respect to attitudes towards HIV testing has not been explored. One study investigating individual differences in discomfort with uncertainty illustrated that individuals with a high need for cognitive closure were more likely to be screened for cervical cancer due to their dispositional preference for an answer and relative discomfort with uncertainty [19]. However, a more recent study suggested that one's need for cognitive closure was negatively associated with lower odds of receiving a flu vaccination [20]. Given mixed findings on the role of cognitive closure in determining health behaviors, this study seeks to examine how the need for closure affects one's attitudes and overall willingness to receive HIV testing.

The goals of the present study are to: 1) investigate inter-correlations between cultural mistrust, medical mistrust, HIV conspiracy beliefs and need for cognitive closure; 2) investigate the relationship between age, cultural mistrust, medical mistrust, HIV conspiracy beliefs, need for cognitive closure and attitudes towards HIV testing; and 3) explore the extent to which cultural mistrust, medical mistrust, HIV conspiracy beliefs and need for cognitive closure predict attitudes towards HIV testing.

We hypothesize that there will be significant inter-correlations between cultural mistrust, medical mistrust, HIV conspiracy beliefs and need for cognitive closure and that these variables will be significantly correlated with age. In addition, we expect that the aforementioned variables will significantly predict an individual's attitudes towards HIV testing. Given the disproportionate burden experienced by Black patients in HIV/AIDS settings, it is imperative that we understand the ways in which cultural mistrust, medical mistrust, conspiracy beliefs and NFCC affect attitudes towards HIV testing. It is our hope that the findings from this study will help to guide prevention and intervention efforts, promote more positive health attitudes and reduce health disparities within the Black community.

\section{Method}

\section{Participants}

The sample was comprised of 118 individuals including undergraduate students recruited from a private, historically Black university and a community college in the mid-Atlantic area and adults recruited from local churches and health forums in the mid-Atlantic area. With an alpha level of 0.05 , statistical power of 0.8 , medium effect size

\begin{tabular}{|l|c|c|c|}
\hline \multicolumn{1}{|c|}{ Variable } & M (SD) & Range & $\boldsymbol{n}(\%)$ \\
\hline Age & 26.8 & $16-67$ & \\
\hline Gender & & & $34(28.8)$ \\
\hline Male & & & $84(71.2)$ \\
\hline Female & & & $67(56.8)$ \\
\hline Prior HIV testing & & $51(43.2)$ \\
\hline Yes & & & $107(90.7)$ \\
\hline No & & $11(9.3)$ \\
\hline Willingness to receive HIV testing & & \\
\hline Yes & & & $114(96.6)$ \\
\hline No & & $4(3.4)$ \\
\hline Place of Birth & & & $113(95.8)$ \\
\hline US-Born & & $5(4.2)$ \\
\hline Non US-Born & & & \\
\hline Majority of life & & & \\
\hline In USA & & & \\
\hline Outside the USA & & & \\
\hline
\end{tabular}

Table 1: Major demographic variable characteristics $(n=118)$.

of 0.15 , and 4 predictors, we expected to need at least 84 subjects. The average participant was 26.83 years old and, among the 118 participants, $84(71.2 \%)$ were female. Although 107 (90.7\%) participants reported being willing to test for HIV, only 67 (56.8\%) reported prior HIV testing. The overwhelming majority of the participants was born in the United States and reported spending the majority of their life in the United States. Detailed demographic information is presented in Table 1.

\section{Measures}

\section{Background questionnaire}

In order to assess demographic information, the Demographic Questionnaire (Appendix A) was administered to participants in the survey. It contained items including gender, age, race/ethnicity and previous HIV testing behaviors.

\section{HIV conspiracy beliefs}

HIV conspiracy-related beliefs were assessed by asking participants to indicate their level of agreement with 5 statements (Appendix B) concerning the origin of HIV/AIDS in the population (e.g. "HIV was released on purpose by the government"); the withholding of a cure for HIV/AIDS (e.g. "The government has a cure for HIV but will not release it"); and the withholding of a vaccine to prevent HIV infection (e.g." "The drug companies have a vaccine to prevent HIV infection but will not release it"). Each question was rated on a 6-point Likert scale (1=strongly disagree, $6=$ strongly agree). An HIV conspiracy beliefs total score was then created to determine the intensity of the belief; this score was defined as the sum of responses to the five conspiracy items, with higher scores indicating greater levels of agreement with HIV conspiracy beliefs. The five conspiracy items have been shown to function well as a scale, with a standardized Cronbach's $\alpha$ of 0.91 [21].

\section{Medical mistrust index}

Medical mistrust is the tendency to distrust institutions of medicine, including medical personnel and clinicians who represent the dominant culture, as a result of past and present discrimination, racial persecution and various medical conspiracy theories [22]. In the current study, medical mistrust was assessed using the Medical Mistrust Index (MMI) [23] (Appendix C), a 17-item scale, which uses a 4-point Likert scale with the following response codes: 1=strongly disagree, $2=$ disagree, $3=$ agree and $4=$ strongly agree. Examples of items included in the MMI 
are: "Patients have sometimes been deceived or mislead by healthcare organizations and "When healthcare organizations make mistakes they usually cover it up." Six items $(3,9,11,13,14,17)$ were reverse coded and a simple summation yielded a MMI total score, where higher totals were indicative of greater medical mistrust. The MMI was used in the present study due to its adequate reliability (Chronbach's $\alpha=0.76$ ).

\section{Cultural mistrust inventory}

The Cultural Mistrust Inventory (CMI) (Appendix D) was developed by Terrell and Terrell and measures Blacks' mistrust of Whites at the cultural level [8]. The measure is based on the notion that cultural mistrust exists in Black culture due to a history of racism, oppression and human rights violations [24]. The CMI is a 48 -item instrument with a 9 -point likert scale $(0=$ not in the least agree, $9=$ entirely agree) and measures cultural mistrust in four distinct areas: politics and law (e.g. "White politicians will promise blacks a lot but deliver little"), interpersonal relations (e.g. "White friends are least likely to break their promise"), education and training (e.g. "Black persons should teach their children not to trust white teachers"), and business and work (e.g. "A Black person can usually trust his or her white co-workers"). Twenty-one items $(1,6,10,12,14,17,19,23,26-28,31-36,41,43,44$, 48) were reverse coded and a simple summation yielded a CMI total score, where higher totals were indicative of greater cultural mistrust.

The CMI was used in this current study because of its strong psychometric properties. Although the external validity of the scale has been questioned [8] reported that the CMI has a test-retest reliability of 0.83 as well as good concurrent, predictive and criterion-related validity. In addition, the CMI has demonstrated good internal consistency with a Chronbach's $\alpha=0.89$ [25]. More recent psychometric analyses confirm the strong reliability and validity of the CMI and factor analytic results indicate that a single global dimension underlies the CMI total score.

\section{Need for cognitive closure}

The need for cognitive closure (NFCC) was assessed by the Need for Closure Scale (NFCS) [26] (Appendix E). The instrument consists of 14 items (e.g. I don't like situations that are uncertain; I'd rather know bad news than stay in a state of uncertainty) rated on a six-point Likert scale ( $1=$ strongly disagree, $6=$ strongly agree) and includes two dummy questions that serve as a measure of validity ("I have never been late for work or an appointment", "I have never hurt another person's feelings"). This scale was scored by totaling the responses to the 14 target statements. Higher scores indicated higher need for closure.

\section{HIV-antibody testing attitude scale}

Attitudes towards HIV testing were measured using the 22-item HIV-Antibody Testing Attitude Scale (HTAS) [27] (Appendix F). The HTAS consists of five subscales or factors. Factor 1: Trust and support about HIV antibody testing (6 items); Factor 2: General concerns about HIV antibody testing (6 items); Factor 3: Fears about HIV antibody testing (4 items); Factor 4: Concerns about confidentiality of HIV antibody testing (3 items) and Factor 5: Friends concerns about HIV antibody testing ( 3 items). For each of the attitude items (e.g. "My friends would look down on me if I were tested for HIV"), participants indicate on a 5-point Likert scale whether they strongly agreed (5), agreed (4), were neutral (3), disagreed (2) or strongly disagreed (1). Strong agreement with an item was given a score of 5 , and strong disagreement was given a score of 1 . Reverse scoring was used for barrier items (e.g. "People would assume I have HIV if I decided to get tested.") Item scores were summed and high scores indicated a more favorable attitude toward HIV testing and a greater willingness to receive HIV testing. Research indicates that the HTAS demonstrates good reliability (Cronbach's $\alpha=0.84$ [27]).

\section{Procedure}

Participants in the undergraduate sample were recruited from the Psychology Department introduction to Psychology human participant pool. Inclusionary criteria included: (a) young adults enrolled in a 4 year college/university, (b) those of Black/African descent and (c) those currently living in the Washington, D.C. area. Additional participants were recruited from a health fair on a community college campus. Participants in the community sample were recruited from local churches and health forums. Inclusionary criteria included: (a) young adults ages 21 and older, (b) those of Black/African descent and (c) those currently living in the Washington, D.C. area.

Participants were informed of the purpose of the study, the anonymity and confidentiality of their responses. After having been given an opportunity to ask questions, participants were asked to sign and return the consent form before completing the demographics questionnaire, followed by questionnaires measuring HIV-related conspiracy beliefs, cultural mistrust, medical mistrust, need for cognitive closure and attitudes towards HIV testing. Participants were advised that the completion of the questionnaires would take approximately 20-30 min. Only paper surveys were used to ensure confidentiality of the participants. Participant anonymity was further protected by numbering each survey sequentially; thus, participants never had to provide their names on the actual survey. Storage of responses and separate signed consent forms were secured in a locked file cabinet, and all electronic files used for storing or processing data were stored in password-protected devices.

All undergraduate participants from the Psychology Department participant pool completed the paper surveys in small groups while they were seated in a private research space. Undergraduate participants from the community college completed the surveys independently at the researcher's booth during the health fair to ensure privacy and confidentiality. Participants in the community sample were also administered the surveys individually and were asked to fill out and return the survey to the research upon completion.

This study was approved by the University's Institutional Review Board (IRB). The risk involved in this study was deemed minimal and none of the undergraduate participants reported discomfort or adverse effects. However, 3 of the older participants in the community sample expressed some discomfort about the content of some the questions, particularly those on the Cultural Mistrust Index. Given that this discomfort was psychological in nature, participants were encouraged to terminate or pause their participation until they felt comfortable enough to resume completion of the survey. In rare situations in which participants were unable to quickly recover from any psychological distress or discomfort, the researchers would have provided referral and contact information for psychological services, such as the University Counseling Center, or other self-help materials as appropriate. To date, no such psychological referrals have been made and there have been no reports of extreme psychological effects from participation in the study.

\section{Results}

\section{Demographic characteristics}

The samples consisted of 118 participants recruited from a private HBCU, a community college, local churches and health forums in the mid-Atlantic area. The participants ranged in age from 16-67 years 
( $\mu=26.8$ years) and consisted of $28.8 \%$ males $(n=34)$ and $71.2 \%$ females $(\mathrm{n}=84)$. Among the participants, $56.8 \%(\mathrm{n}=67)$ reported having had an HIV test in the past and $43.2 \%(\mathrm{n}=51)$ denied ever being testing. Of those who received an HIV test, 100\% reported being HIV negative. In addition, $90.7 \%(n=107)$ reported being willing to get tested in the future while $9.3 \%(n=11)$ reported an unwillingness to be tested in the future.

\section{Hypotheses testing}

We hypothesized that there would be significant correlations between cultural mistrust, medical mistrust, HIV conspiracy beliefs, need for cognitive closure, age and attitudes towards HIV testing. In order to assess these relationships, correlation analyses were used; these correlations are displayed in Table 2. As predicted, cultural mistrust was positively correlated with medical mistrust and HIV conspiracy beliefs. In other words, as cultural mistrust increased, levels of medical mistrust and HIV conspiracy beliefs increased also. However, contrary to our hypothesis, the need for cognitive closure was not positively correlated with conspiracy theories. Instead, need for cognitive closure was positively correlated with medical mistrust. Furthermore, none of the variables was significantly correlated with the major dependent variable, attitude towards HIV testing.

When looking at the individual factors latent within the HTAS measure of attitudes towards HIV testing, there was a significant negative correlation between cultural mistrust, medical mistrust and Factor 4 (Concerns about confidentiality of HIV antibody testing). Additionally, there was a significant negative correlation between Factor 5 (Friends concerns about HIV antibody testing) and age.

Results also indicated that there was no significant correlation between age, cultural mistrust, medical mistrust and willingness to receive HIV testing. However, there was a significant negative correlation between age and the need for cognitive closure as well as age and HIV conspiracy beliefs (Figures 1 and 2). In other words, as individuals increase in age, they have a lesser need for cognitive closure and are less likely to hold HIV conspiracy beliefs.

Given that none of the predicted variables (cultural mistrust, medical mistrust, HIV conspiracy theories, need for cognitive closure) were significantly correlated with the major dependent variable (attitudes towards HIV testing) it was not surprising that the first multiple regression model using these four variables was not predictive of attitudes towards HIV testing (Table 3 ).

Given that age was associated with the need for cognitive closure and HIV conspiracy beliefs, we controlled for age in the second multiple regression models. After controlling for age, the model was still not significant and age did not add to the predictive ability of the model (Table 4).

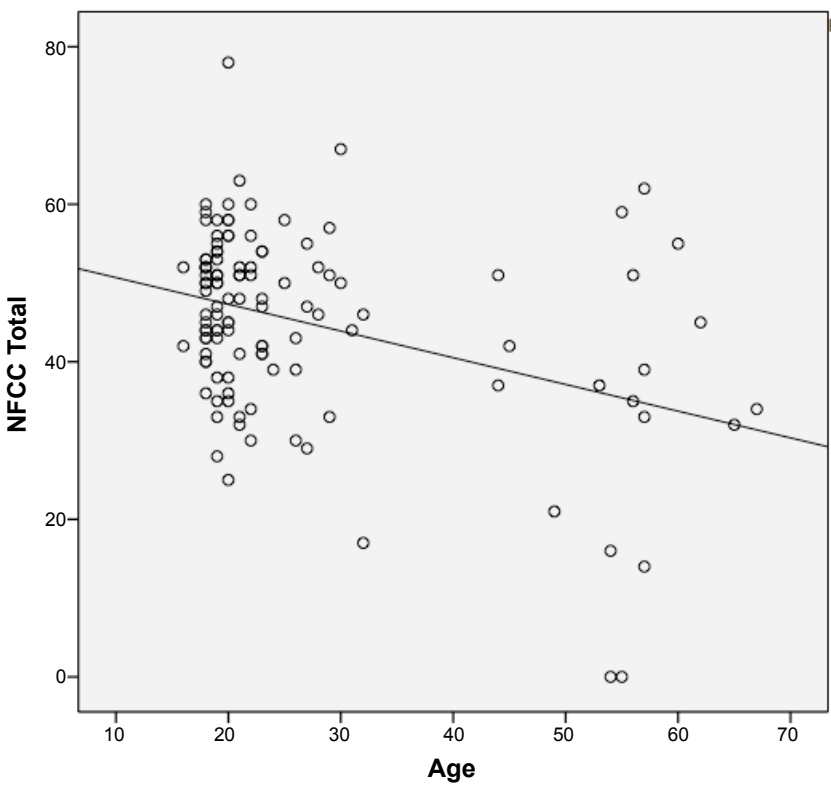

Figure 1: Correlation between age and the need for cognitive closure.

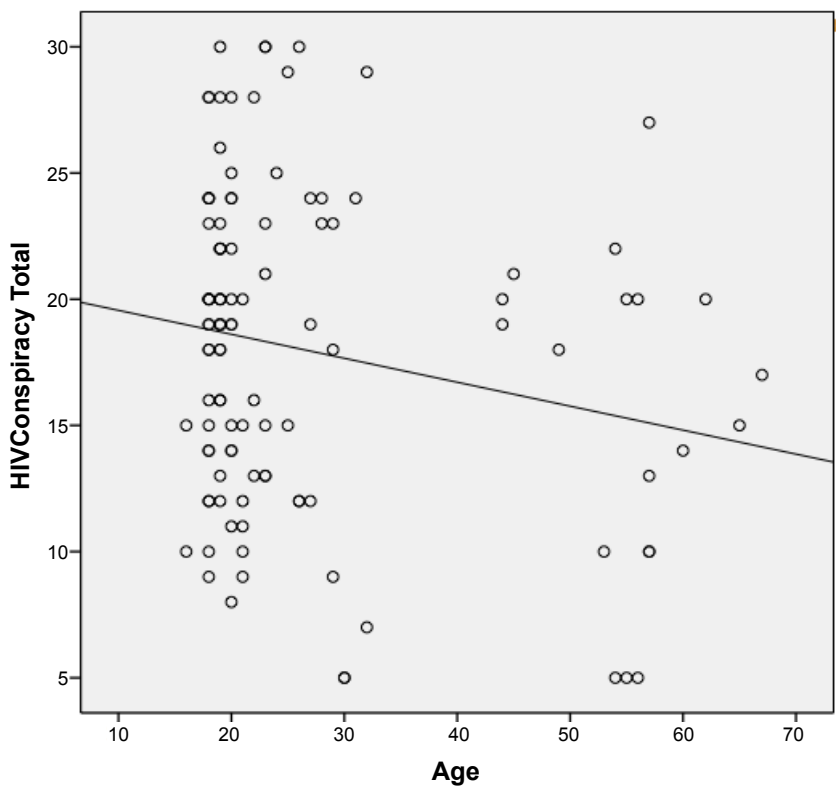

Figure 2: Correlation between age and HIV conspiracy beliefs.

\begin{tabular}{|c|c|c|c|c|c|c|c|c|}
\hline & $\begin{array}{l}\text { Cultural } \\
\text { Mistrust }\end{array}$ & $\begin{array}{l}\text { Medical } \\
\text { Mistrust }\end{array}$ & $\begin{array}{l}\text { HIV Conspiracy } \\
\text { Beliefs }\end{array}$ & $\begin{array}{l}\text { Need for } \\
\text { Cognitive } \\
\text { Closure }\end{array}$ & $\begin{array}{l}\text { Attitude Towards } \\
\text { HIV Testing }\end{array}$ & Age & HTAS Factor 4 & HTAS Factor 5 \\
\hline Cultural Mistrust & 1 & $0.42^{\star \star}$ & $0.48^{\star \star}$ & -0.10 & -0.15 & -0.10 & $-0.20^{\star \star}$ & -0.08 \\
\hline Medical Mistrust & $0.42^{* \star}$ & 1 & $0.24^{*}$ & $0.21^{*}$ & -0.06 & -0.15 & $-0.25^{\star *}$ & -0.15 \\
\hline HIV Conspiracy Beliefs & $0.48^{\star *}$ & $0.24^{*}$ & 1 & 0.01 & 0.06 & $-.20^{*}$ & -0.09 & 0.03 \\
\hline Need for Cognitive Closure & -0.10 & $0.21^{*}$ & 0.01 & 1 & 0.11 & $-0.37^{\star *}$ & 0.04 & 0.11 \\
\hline Attitude Towards HIV Testing & -0.15 & -0.06 & 0.06 & 0.11 & 1 & -0.13 & $0.71^{* *}$ & $0.69^{* \star}$ \\
\hline Age & -0.10 & -0.15 & $-.20^{*}$ & $-0.37^{* *}$ & -0.13 & 1 & -0.02 & $-0.20^{*}$ \\
\hline HTAS Factor 4 & $-0.20^{* \star}$ & $-0.25^{\star \star}$ & -0.09 & 0.04 & $0.71^{* *}$ & -0.02 & 1 & $0.37^{\star \star}$ \\
\hline HTAS Factor 5 & -0.08 & -0.15 & 0.03 & 0.11 & $0.69^{* *}$ & $-0.20^{*}$ & $0.37^{* *}$ & 1 \\
\hline
\end{tabular}

Table 2: Correlation matrix of cultural mistrust, medical mistrust, HIV conspiracy beliefs, need for cognitive closure, attitudes towards HIV testing, age, HTAS Factors 4 and 5 . 


\begin{tabular}{|l|c|c|c|}
\hline Variable & B & SE $\boldsymbol{\beta}$ & $\boldsymbol{\beta}$ \\
\hline Cultural Mistrust & -0.064 & 0.033 & -0.222 \\
\hline Medical Mistrust & -0.053 & 0.241 & -0.024 \\
\hline HIV Conspiracy Beliefs & 0.360 & 0.247 & 0.163 \\
\hline Need for Cognitive Closure & 0.092 & 0.124 & 0.072 \\
\hline
\end{tabular}

Table 3: Multiple regression model for attitudes towards HIV testing.

\begin{tabular}{|l|c|c|c|}
\hline Variable & B & SE $\boldsymbol{\beta}$ & $\boldsymbol{\beta}$ \\
\hline Cultural Mistrust & -0.066 & 0.034 & -0.231 \\
\hline Medical Mistrust & -0.045 & 0.241 & -0.020 \\
\hline HIV Conspiracy Beliefs & 0.324 & 0.249 & 0.147 \\
\hline Need for Cognitive Closure & 0.045 & 0.132 & 0.036 \\
\hline Age & -0.115 & 0.113 & -0.107 \\
\hline
\end{tabular}

Table 4: Multiple regression model for attitudes towards HIV testing (controlling for Age).

\begin{tabular}{|l|c|c|c|c|c|c|}
\hline Variable & B & SE & Wald & df & Sig. & Exp (B) \\
\hline Cultural Mistrust & 0.007 & 0.008 & 0.744 & 1 & 0.388 & 1.007 \\
\hline Medical Mistrust & -0.063 & 0.061 & 1.055 & 1 & 0.304 & 0.939 \\
\hline HIV Conspiracy Beliefs & -0.036 & 0.067 & 0.286 & 1 & 0.593 & 0.965 \\
\hline Need for Cognitive Closure & 0.038 & 0.032 & 1.378 & 1 & 0.240 & 1.039 \\
\hline
\end{tabular}

Note: Dependent variable, willingness to receive HIV testing, coded such that $0=$ not willing, $1=$ willing

Table 5: Logistic regression model for willingness to receive HIV testing.

Auxiliary analyses were conducted, and a logistical regression was performed to ascertain the effects of cultural mistrust, medical mistrust, HIV conspiracy theories, need for cognitive closure on one's subjective willingness to get tested (Yes/No). Logistical regression revealed that this model was also not significant (Table 5).

\section{Discussion}

In the present study, there were a number of surprising findings. First, none of the variables was significantly correlated with the major dependent variable, attitude towards HIV testing. These findings are contrary to the literature suggesting that cultural mistrust, medical mistrust and conspiracy theories are associated with lower odds of HIV testing [28]. There are a number of possible explanations for this lack of correlation. Firstly, the HTAS measure may have been limited in its ability to fully capture an individual's attitudes towards HIV testing. Currently, no study has tested the construct validity of the HTAS, and this may be an important goal of future research. Secondly, attitudes towards HIV testing among the participants were skewed, resulting in a pronounced ceiling effect, which certainly may have impacted the results. On the HTAS, the average score was 92.83 , on a scale of 22 110; this indicates a relative willingness to receive testing among the sample participants. Figure 3 below illustrates the spread of the data, and highlights a potential reason for the lack of correlation between the major independent variables and the dependent variable.

In addition, it is possible that there were other variables that were more directly correlated with attitudes towards HIV testing, and that cultural mistrust, medical mistrust, conspiracy beliefs and NFCC acted as mediators and/or moderators instead. Overall, this finding is significant in the sense that it suggests that there may be other, more relevant and influential determinants of willingness to get tested among Blacks, which will be considered further in the discussion of future directions.

A second surprising finding was the significant negative correlation between age and the need for cognitive closure as well as age and HIV conspiracy beliefs. Given the neurocognitive research suggesting that

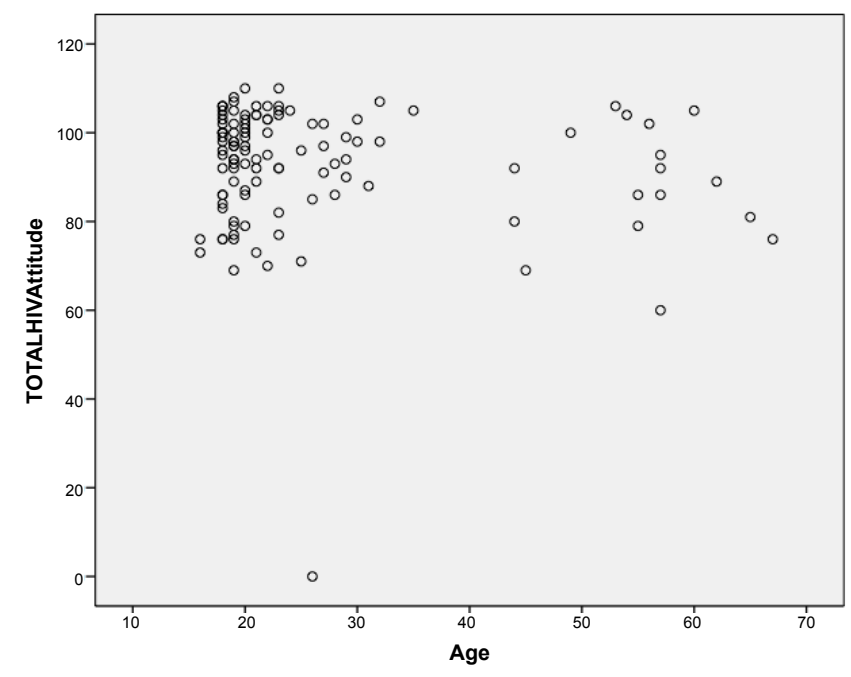

Figure 3: Spread of HIV attitude.

conspiracy theories may form in reaction to distrustfulness, paranoia, low self-esteem and powerlessness [29], it was hypothesized that older Blacks, who have experienced institutional discrimination would be more motivated for closure and more likely to believe an HIV conspiracy theory. One explanation for the negative correlation between age and need for cognitive closure may be found in research suggesting that an individual's tolerance for ambiguity actually increases over time and with experience that comes with age [30]. Similarly, given the fact that the majority of the younger individuals in the sample were from HBCUs, they may have been more aware of historical injustices and discrimination against Blacks. Their Black identity may have been strong, resulting in a greater tendency to believe conspiracy theories as a way to attribute blame to an unjust social system [31].

Despite these surprising findings, a significant positive correlation was found between cultural mistrust, medical mistrust and HIV conspiracy beliefs. In other words, as cultural mistrust increased, levels of medical mistrust and HIV conspiracy beliefs increased also. This finding is similar to other research that supports the interconnectedness of these variables [10].

\section{Limitations}

While the overarching goal of this study was to determine the factors that best explain attitudes towards HIV testing in the Black community, we did not utilize a sample from the general population. Instead, a convenience sample of college-students at a private $\mathrm{HBCU}$, a community college and older adults at local churches and health fairs in the mid-Atlantic area was used. In addition, all of the participants were from the Washington, D.C. area. For reasons of feasibility and relative convenience, these sample populations were chosen. The sampling method poses an issue for generalizability. As such, results of this study should be interpreted with caution, as the results may not be generalizable to all or even most of the Black population in the country.

Additionally, over $50 \%$ of the participants in our sample were under the age of 21 and over $80 \%$ were under the age of 30 . The fact that a disproportionate number of individuals were young adults may not only influence the generalizability of the results to the general Black population, but it also may influence the levels of mistrust and overall attitudes towards HIV observed in this population. According to our hypotheses, younger Black adults have fewer experiences with 
discrimination than older Black adults and, therefore, may report less cultural mistrust. Given that the majority of our population was comprised of younger adults, this may have significantly affected the independent variables.

In this study, the majority of our participants $(71.2 \%)$ were Black females. Females may experience different levels of cultural mistrust, medical mistrust, HIV conspiracy beliefs and need for cognitive closure than Black males. These gender differences may have impacted our results; however, the effect on the study outcome is unknown. Recruiting more men and as well as more older participants certainly could have improved the robustness of the study and may have, potentially, produced different results.

Furthermore, the issue of social desirability may have affected the results, and the study may have benefitted from the inclusion of a social desirability scale or a measure detecting participant's truthfulness. Given that HIV is a significant public health concern in the DC area, participants may have felt unconcious pressure to report favorable attitudes towards testing. This may provide one explanation for the skewed results in the dependent variable (attitudes towards HIV testing).

Other variables that we could not control for included socioeconomic status (SES), education levels, religion and culture. Given that none of our independent variables correlated with or were consistently predictive of attitudes towards HIV testing, it raises the question of the role of other variables in determining one's attitude towards HIV testing [32].

\section{Implications}

Our results have clear implications for the design and delivery of HIV services, prevention interventions and public health educational campaigns. Although none of our major variables was related to attitudes towards HIV testing, the study did highlight a potential disconnect between HIV testing attitudes and behavior.

The results of this study indicated that the overwhelming majority (91\%) of participants stated that they would be willing to receive HIV testing. This indicates a degree of openness towards the idea of HIV testing among Blacks in the sample. However, only $57 \%$ of the sample reported prior HIV testing. Research in this area paints a similar picture. In some studies, as few as $45 \%$ of individuals ages 18-65 reported ever being tested for HIV [33]. The fact that individuals want to know their status and are open to receive testing is often overshadowed by a greater fear of learning the results. This fear of the facts results in avoidance and general ambivalence for many African Americans [34]. It will be important for public health to address this gap between attitudes and behaviors by increasing availability of testing and also targeting the psychological component of testing.

This research also highlights the fact that attitudes towards HIV testing may not be the major issue with regard to HIV prevention efforts. Rather, there may be other psychological and logistical barriers that undermine prevention and intervention efforts.

As was found in our study, cultural mistrust was significantly associated with medical mistrust; medical mistrust has been cited as a major barrier to HIV testing in clinical practice [35]. In order to address health disparities with issues such as HIV, clinicians must address medical mistrust. In therapeutic interventions such as Cognitive Behavioral Therapy (CBT), clinicians can explore the impact of transgenerational traumas, such as racism and racial oppression.
During therapy, clinicians can also address the conspiracy beliefs and HIV/AIDS "denialism" that seems to plague various subgroups within the Black community. In this study, younger individuals were more likely to believe conspiracy beliefs, which have the potential to evolve into HIV denialism. Research suggests that HIV denialism has directly inflicted harm to individuals who avoid getting tested and are dissuaded from seeking treatment for their HIV infection [36].

Finally, it is imperative that clinicians build sincere and genuine rapport with the Black community. Building trusting relationships with health care providers may help to counterbalance much of the mistrust that fuels denialism. By creating trust and assurance that the medical community's intentions are to in fact decrease health disparities and provide safe medical services, Blacks may be more open to receiving treatment for various medical conditions and engage in preventative and intervention efforts for communicable diseases like HIV.

Future studies should look into the role of mistrust, conspiracy beliefs and NFCC in more ethnically diverse Black populations as well as other high-risk populations such as minority men who have sex with men (MSM), African American females, IV users and sex workers. It is also possible that there are personality variables at play that may influence attitudes towards HIV testing [37] and these variables should be explored in future research. Social variables such as social status and education attainment have been linked to frequency of HIV testing [32] and future research should certainly include these variables in addition to the aforementioned variables in determining their impact on attitudes among minority populations.

\section{Conclusion}

Research has consistently demonstrated that early detection and treatment can dramatically reduce HIV-related illnesses, lessen risk of premature death, and maintain health and quality of life over sustained periods [34]. As such, HIV public health agendas are now, more than ever, pushing to increase awareness of the benefits of HIV testing and treatment. Given the fact that decades of surveillance studies consistently show higher HIV incidence and prevalence among African American MSM than among MSM of other races and ethnicities [38], it is clear that a disproportionate HIV burden is carried by people of color. Research suggests that cultural mistrust, medical mistrust an HIV conspiracy theories play an integral role in HIV-related behaviors [10]; however, research on the roles of these factors in determining attitudes towards HIV testing has been lacking.

This study replicated a number of interesting findings present in the literature regarding the relationship between cultural mistrust, medical mistrust an HIV conspiracy theories. In addition, this study introduced a cognitive variable, the need for cognitive closure and demonstrated its relationship to medical mistrust. Although cultural mistrust, medical mistrust, HIV conspiracy theories and need for cognitive closure were not significant predictors of attitudes towards HIV testing, this study highlighted the need for further exploration of factors contributing to attitudes towards testing. Furthermore, the findings of this study shed light on the discrepancy or gap between attitudes towards HIV testing and actual testing behavior. Although many Blacks report a willingness to receive testing, many of them fail to follow through. It is this gap between attitude and behavior that research, public health experts and clinicians must address.

\section{References}

1. Kalichman SC, Hunter TL (1993) HIV-related risk and antibody testing: an urban community survey. AIDS Educ Prev 5: 234-243. 
Citation: Ball K (2016) Cultural Mistrust, Conspiracy Theories and Attitudes towards HIV Testing Among African Americans. J AIDS Clin Res 7: 602. doi:10.4172/2155-6113.1000602

2. Centers for Disease Control and Prevention (2015) Monitoring selected national HIV prevention and care objectives by using HIV surveillance dataUnited States and 6 dependent areas-2013. HIV Surveillance Supplemental Report 20.

3. Friedman SR, Cooper HL, Osborne AH (2009) Structural and social contexts of HIV risk Among African Americans. Am J Public Health 99: 1002-1008.

4. Centers for Disease Control and Prevention (2012) HIV Among African Americans. CDC

5. Xia Q, Nonoyama A, Molitor F, Webb D, Osmond D (2011) Recent decline in the incidence of human immunodeficiency virus infection among California men who have sex with men. American Journal of Epidemiology 174: 203-210.

6. Shelton RC, Winkel G, Davis SN, Roberts N, Valdimarsdottir H (2010) Validation of the group-based medical mistrust scale among urban black men. Journal of General Internal Medicine 25: 549-555.

7. Armstrong K, Putt M, Halbert CH, Grande D, Schwartz JS et al. (2013) Prior experiences of racial discrimination and racial differences in health care system distrust. Med Care 51: 144-150.

8. Terrell R, Terrell SL, Taylor J (1981) Effects of race of examiner and cultura mistrust on the WAIS performance of Black students. Journal of Consulting and Clinical Psychology 49: 750-751.

9. Thompson CE, Neville N, Weathers PL, Poston WC, Atkinson DR (1990) Cultural mistrust and racism reaction among African American students. Journal of College Student Development 31: 162-168.

10. Bogart LM, Galvan FH, Wagner GJ, Klein DJ (2011) Longitudinal association of HIV conspiracy beliefs with sexual risk among black males living with HIV. AIDS Behav 15: 1180-1186.

11. Beach M, Keruly J, Moore R (2006) Is the quality of the patient-provide relationship associated with better adherence and health outcomes for patients with HIV? Journal of General Internal Medicine 21: 661-665.

12. Gaston GB, Alleyne-Green B (2013) The impact of African Americans' beliefs about HIV medical care on treatment adherence: A systematic review and recommendations for interventions. AIDS Behav 17: 31-40.

13. Washington HA (2008) Medical apartheid: The dark history of medical experimentation on Black Americans from colonial times to the present.

14. Mugavero MJ, Lin HY, Allison JJ, Willig JH, Chang PW, et al. (2007) Failure to establish HIV care: characterizing the "no show" phenomenon. Clin Infect Dis 45: $127-130$.

15. Casagrande SS, Gary TL, LaVeist TA, Gaskin DJ, Cooper LA (2007) Perceived discrimination and adherence to medical care in a racially integrated community. Society of General Internal Medicine 22: 389-395.

16. Ross MW, Essien EJ, Torres I (2006) Conspiracy beliefs about the origin of HIVIAIDS in four racial/ethnic groups. Journal of Acquired Immune Deficiency Syndrome 41: 342-344.

17. Dahl V, Mellhammar L, Bajunirwe F, Björkman P (2008) Acceptance of HIV testing among women attending antenatal care in south-western Uganda: Risk factors and reasons for test refusal. AIDS Care 20: 746-752.

18. Kruglanski AW (1990) Motivations for judging and knowing: Implications for social attributions. Handbook of motivation and cognition: Foundations of social behavior, New York: Guilford 2: 335-368.

19. Eiser JR, Cole N (2002) Participation in Cervical Screening as a Function of Perceived Risk, Barriers and need for Cognitive Closure. J Health Psychol 7: 99-105.

20. Cole AP, Gill JM, Fletcher KD, Shivers CA, Allen LC, et al. (2015) Understanding African American college students' H1N1 vaccination decisions. Health Psychology 34: 1185
21. Clark A, Mayben JK, Hartman C, Kallen MA, Giordano TP (2008) Conspiracy beliefs about HIV infection are common but not associated with delayed diagnosis or adherence to care. AIDS Patient Care STDS 22: 753-759.

22. Ball K, Lawson W, Alim T (2013) Medical mistrust, conspiracy beliefs and HIV related behavior among African Americans. Journal of Psychology and Behavioral Science 1.

23. LaVeist TA, Nickerson KJ, Bowie JV (2000) Attitudes about racism, medica mistrust and satisfaction with care among African American and white cardiac patients. Medical Care Research and Review 57: 146-161.

24. Cobb PM, Grier W (1968). Black rage, New York: Bantam.

25. Nickerson KJ, Helms JE, Terrell F (1994) Cultural mistrust, opinions about mental illness and Black students' attitudes toward seeking psychological help from counselors. Journal of Counseling Psychology 41: 378-385.

26. Webster DM, Kruglanski AW (1994) Individual differences in need for cognitive closure. J Pers Soc Psychol 67: 1049-1062.

27. Peltzer K, Mpofu E (2002) The factor structure of the HIV attitude scale in fou African countries. Eastern Journal of Medicine 7: 27-30.

28. Hoyt MA, Rubin LR, Nemeroff CJ, Lee J, Huebner DM, et al. (2012) HIVIAIDSrelated institutional mistrust among multiethnic men who have sex with men: Effects on HIV testing and risk behaviors. Health Psychol 31: 269-277.

29. Koerth-Baker M (2013) Sure you saw a flying saucer. NY Times Magazine MM15.

30. Endres ML, Camp R, Milner M (2015) Is ambiguity tolerance malleable? Experimental evidence with potential implications for future research. Frontiers in psychology 6

31. Crocker J (1999) Social stigma and self-esteem: Situational construction of self-worth. Journal of Experimental Social Psychology 35: 89-107.

32. Mitchell JW, Horvath KJ (2013) Factors associated with regular HIV testing among a sample of US MSM with HIV-negative main partners. J Acquir Immune Defic Syndr 64: 417-423.

33. Johnson AS, Heitgerd J, van Koenig LJHM, Branson BM, Connelly E, et al (2010) Vital signs: HIV testing and diagnosis among adults-United States, 2001-2009. Morbidity and Mortality Weekly Report 59: 1550-1555.

34. St. Lawrence JS, Kelly JA, Dickson-Gomez J, Owczarzak J, Amirkhanian YA, et al. (2015) Attitudes toward HIV voluntary counseling and testing (VCT) among African American men who have sex with men: Concerns underlying reluctance to test. AIDS Education and Prevention 27: 195-211.

35. Daniels P, Wimberly Y (2004) HIV testing rates among African Americans: Why are they not increasing? J Natl Med Assoc 96: 1107-1108.

36. Chigwedere P, Essex M (2010) AIDS denialism and public health practice. AIDS Behav 14: 237-247.

37. Swami V (2012) Social psychological origins of conspiracy theories: The case of the Jewish conspiracy theory in Malaysia. Front Psychol 3: 280.

38. Balaji AB, Bowles KE, Le BC, Paz-Bailey G, Oster AM, et al. (2013) High HIV incidence and prevalence and associated factors among young MSM, 2008. AIDS 27: 269-278. 